First publ. in: Physical Review Letters 88 (2002), Article 055702

\title{
Triple-Point Wetting on Rough Substrates
}

\author{
A. Esztermann, M. Heni, and H. Löwen \\ Institut für Theoretische Physik II, Heinrich-Heine-Universität Düsseldorf, Universitätsstrasse 1, 40225 Düsseldorf, Germany
}

\author{
J. Klier, M. Sohaili, and P. Leiderer \\ Fakultät für Physik, Universität Konstanz, Postfach 5560 M675, 78457 Konstanz, Germany
}

(Received 21 June 2001; published 18 January 2002)

\begin{abstract}
The influence of substrate roughness on the wetting scenario of adsorbed van der Waals films is investigated by theory and experiment. Calculating the bending free energy penalty of a solid sheet picking up the substrate roughness, we show that a finite roughness always leads to triple-point wetting reducing the widths of the adsorbed solid films considerably as compared to that of smooth substrates. Testing the theory against our experimental data for molecular hydrogen adsorbed on gold, we find quantitative agreement.
\end{abstract}

PACS numbers: $64.70 . \mathrm{Hz}, 67.70 .+\mathrm{n}, 68.08 . \mathrm{Bc}, 68.35 . \mathrm{Rh}$

Wetting of a solid substrate, exposed to a gas in thermodynamic equilibrium, is an ubiquitous phenomenon, with both fundamental aspects [1,2] and important applications [3-5]. Microscopically, substrate wetting by a liquid film is caused by a strong substrate-particle attraction mediated by van der Waals forces. At present, an almost complete microscopic understanding of wetting on flat solid substrates is available $[1,2,6]$ predicting the thickness of the liquid film as a function of the substrate-particle and interparticle interactions for given thermodynamic parameters such as temperature and pressure. The following basic theoretical predictions were confirmed by experiments using, e.g., noble gases [1] on different substrates: (i) For fixed thermodynamic conditions, the thickness of the wetting layer grows for increasing substrate-particle attraction. (ii) Complete wetting (i.e., a diverging thickness of the liquid layer) occurs if the substrate-particle attraction is stronger than the interparticle interaction and the thermodynamic conditions approach liquid-gas coexistence. The latter condition can be achieved only if the system temperature $T$ is above the triple temperature $T_{3}$. For $T<T_{3}$, on the other hand, a solid film shows up near the sublimation line. Various experiments have shown [7-10] that the width of the solid layer always remains finite when approaching gas-solid coexistence. It is only near the triple point that a liquid layer on top of the solid sheet is formed, with a diverging width as the triple point is approached. This universal behavior is called "triple-point wetting."

One major difference between a liquid and solid wetting layer is that a solid cannot relax the elastic compression caused by the substrate attraction as embodied in the (reduced) wall-particle Hamaker constant $R$. This fact is the basic ingredient in the traditional Gittes-Schick theory [11] of solid adsorption on flat substrates. It predicts that, for a particular value $R=R_{0}$ of the substrate attraction, complete wetting is possible, while for $R>R_{0}$, in contrast to liquid wetting, the thickness of the solid film $\ell_{s}$ decreases with increasing $R$. In this Letter we show that the key parameter governing adsorption of solid films is the substrate roughness rather than the elastic deformation caused by the substrate attraction. As a result of our theoretical analysis, a finite substrate roughness leads inevitably to triple-point wetting and yields, for realistic parameters, a thickness $\ell_{s}$ growing with $R$ in quantitative agreement with our experimental data. As compared to Gittes-Schick theory, which neglects substrate roughness, the film thickness $\ell_{s}$ is considerably reduced even for small roughness. Hence triple-point wetting originates from and is controlled by substrate roughness.

We first describe the theoretical treatment of this problem, and further present, in what follows, a comparison with experiments on a system for which triple-point wetting has been studied rather extensively, namely molecular hydrogen adsorbed on a gold substrate. We calculate, for fixed temperature $T$ and pressure $P$, the excess grand canonical free energy $\Sigma\left(\ell_{s}, \ell_{\ell}\right)$ per unit area relative to a nonwetting situation. This is done for a compound adsorption layer involving a solid sheet of width $\ell_{s}$ on top of the rough substrate and a liquid sheet of width $\ell_{\ell}$ on top of the solid. $\Sigma$ involves three different terms, $\Sigma=\Sigma_{1}+\Sigma_{2}+\Sigma_{3}$, resulting from thermodynamics, from the lateral elastic strain in the solid and from the elastic free energy cost of a distorted solid picking up the substrate roughness. While the first and second terms are familiar from previous considerations for smooth walls, the third term is essential for nonideal substrates. In detail, the thermodynamic part is $[1,12]$

$$
\begin{aligned}
\Sigma_{1}\left(\ell_{s}, \ell_{\ell}\right)= & \gamma_{w s}+\gamma_{s \ell}+\gamma_{\ell g}-\gamma_{w g} \\
& +\rho_{s}\left(P_{0}-P\right) \ell_{s} / \rho_{g}+\rho_{\ell}\left(P_{0}^{\prime}-P\right) \ell_{\ell} / \rho_{g} \\
& +A_{1} / \ell_{s}^{2}+A_{2} / \ell_{\ell}^{2}+A_{3} /\left(\ell_{s}+\ell_{\ell}\right)^{2},
\end{aligned}
$$

where $\gamma_{i j}$ are the extrapolated interfacial tensions between wall $(w)$, solid $(s)$, liquid $(\ell)$, and gas $(g)$, and $P_{0}\left(P_{0}^{\prime}\right)$ is the coexistence pressure between gas and solid (liquid). The number densities at solid-gas coexistence are $\rho_{s}$ and $\rho_{g}$, while $\rho_{\ell}$ is the liquid number density 
at liquid-gas coexistence. We assume $\rho_{g} \ll \rho_{\ell}<\rho_{s}$. In case the coexistence is metastable, these quantities are extrapolated from their thermodynamically stable regions. Furthermore, $A_{1}=\left(\rho_{s}-\rho_{\ell}\right)\left(C-\rho_{s} H\right), A_{2}=$ $\left(\rho_{s}-\rho_{\ell}\right) \rho_{\ell} H$, and $A_{3}=\rho_{\ell}\left(C-\rho_{s} H\right)$ where $C$ and $H$ are Hamaker constants of the van der Waals tails of the particle-substrate and particle-particle interaction potentials decaying as $-2 C / z^{3}$ and $-12 H / \pi r^{6}$ for large separations $z$ or $r$ [1]. Equation (1) is valid for thermodynamic conditions close to the triple point and for thicknesses $\ell_{s}, \ell_{\ell}$ larger than microscopic distances. When setting $\ell_{\ell}=0$ a priori, the theory can also be used away from the triple point provided the pressure is close to the sublimation pressure.

Second, there is a free energy penalty $\Sigma_{2}$ due to solid strain caused by the substrate attraction [7] which reads for large $\ell_{s}$ as [11]

$$
\Sigma_{2}\left(\ell_{s}\right)=-B S^{2} / \ell_{s}-2 B S^{3} / \ell_{s}^{2},
$$

where $B=\frac{3 E}{2(1+\nu)}, E$ is Young's modulus of the adsorbed solid, and $\nu$ is its Poisson ratio. Finally $S=0.0229(R-$ $\left.R_{0}\right) \sigma$ is the total reduced stress, with $\sigma$ denoting a molecular length scale. Furthermore, $R=C / H \rho_{s}$ is the ratio between adsorbate-substrate and adsorbate-adsorbate interaction. The total stress is zero for $R \equiv R_{0}=1.88$. Hence complete wetting is possible only for this particular ratio $R$. Combining $\Sigma_{2}$ with $\Sigma_{1}$, the traditional GittesSchick theory of triple-point wetting is obtained.

While these contributions are essential for smooth substrates, a surface roughness induces an additional bending of the adjacent crystalline sheet [13]. For typical nonideal substrates, the surface modulation varies on a characteristic lateral length scale $b$ which is much larger than its vertical (valley to peak) height $h$, i.e., $b \gg h$. We further assume $b \gg \ell_{s}$ [14]. In more detail, we characterize the surface roughness by a height variable $\zeta(x, y)$ where the substrate lies in the $x y$ plane. Then a weakly bent crystalline layer of width $\ell_{s}$ costs the following elastic free energy per area $A$ [15]:

$$
\Sigma_{3}\left(\ell_{s}\right)=\frac{E \bar{G}}{24\left(1-\nu^{2}\right)} \ell_{s}^{3}
$$

with $\bar{G}$ measuring the strength of roughness

$$
\begin{aligned}
\bar{G}=\frac{1}{A} \iint_{A}\{ & \left(\frac{\partial^{2} \zeta}{\partial x^{2}}+\frac{\partial^{2} \zeta}{\partial y^{2}}\right)^{2} \\
& \left.+2(1-\nu)\left[\left(\frac{\partial^{2} \zeta}{\partial x \partial y}\right)^{2}-\frac{\partial^{2} \zeta}{\partial x^{2}} \frac{\partial^{2} \zeta}{\partial y^{2}}\right]\right\} d x d y .
\end{aligned}
$$

By scaling arguments one obtains $\bar{G} \propto h^{2} / b^{4}$.

The equilibrium solid and liquid thicknesses are obtained by minimizing $\Sigma\left(\ell_{s}, \ell_{\ell}\right)$ with respect to $\ell_{s}$ and $\ell_{\ell}$ resulting in a total thickness $\ell=\ell_{s}+\ell_{\ell}$. Clearly, the bending free energy will prevent complete wetting by a solid sheet and thus enforce triple-point wetting even when the total reduced strain $S$ vanishes. As a result, the finite thickness of $\ell_{s}$ at solid-gas coexistence far away from the triple point scales as $\ell_{s} \propto\left(C-\rho_{s} H\right)^{1 / 5} b^{4 / 5} h^{-2 / 5}$ provided the total reduced stress is small, $S \ll \rho_{s}^{2} C^{2} / E^{2} \bar{G}^{3 / 2}$.

Our experimental data were obtained by surface plasmon (SP) spectroscopy as described earlier [10]. The Au films which served as a substrate in these measurements had a thickness of $450 \AA$ (this thickness provides an optimum in the SP resonance signal) and were usually prepared by thermal evaporation on a glass prism. After evaporation the prism was mounted in a sample cell in an optical ${ }^{4} \mathrm{He}$ flow cryostat. The temperature could be varied between 6 and $90 \mathrm{~K}$ with a resolution and stability of a few $\mathrm{mK}$. Using the SP spectroscopy method gave a resolution of the total thickness $\ell$ of the adsorbed films of about 0.1 monolayer. Our data were taken along two different routes: on one hand, adsorption isotherms were obtained by admitting the hydrogen gas in small steps, while keeping the temperature constant, until the corresponding saturated vapor pressure was reached. On the other hand, temperature scans at (nearly) saturated vapor pressure were taken by admitting sufficient hydrogen gas above $T_{3}$ so that bulk liquid formed at the bottom of the sample cell. Then the temperature was slowly swept substantially below $T_{3}$ and back again. Before taking each data point, enough time was given to ensure equilibrium conditions. The equilibration times were found to differ considerably depending on the thickness of the adsorbed film and the temperature range.

In order to compare our theory to the experimental data, we adopt a Lennard-Jones picture of the hydrogen particles and the gold-hydrogen interaction resulting in [16] $H=2.44 \mathrm{eV} \AA^{6}, C=0.39 \mathrm{eV} \AA^{3}$ with a molecular Lennard-Jones diameter $\sigma=3.04 \AA$, leading to $R=4.5$. The temperature-dependent thermodynamic input parameters such as coexistence pressures and number densities are known from the bulk phase diagram of a Lennard-Jones system [17] and data for the temperature-dependent bulk elastic constants for a Lennard-Jones fcc crystal are also available [18]. The roughness of the substrate is directly measured by atomic force microscopy (AFM); an example is shown in Fig. 1a exhibiting typical values of $h=50 \AA$ and $b=500 \AA$. The roughness $\bar{G}$ is calculated from these data resulting in $\bar{G}=5.4 \times 10^{-4} / \sigma^{2}$.

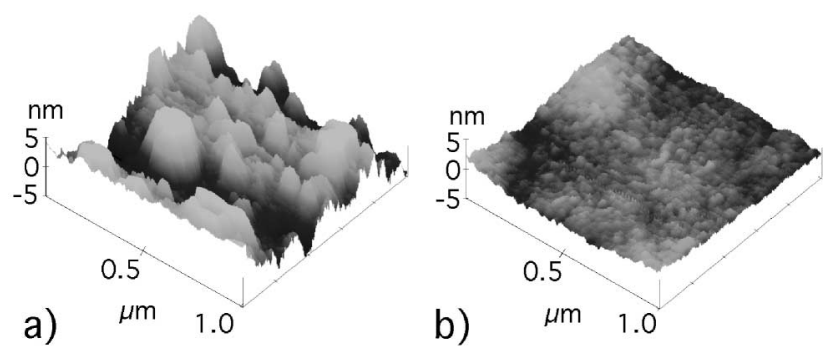

FIG. 1. Surface profile of the rough substrate as measured by AFM: (a) evaporated gold film; (b) gold film detached from a silicon wafer. 
Results for the total width $\ell$ of the adsorbed hydrogen film versus reduced pressure are shown in Fig. 2 for temperatures both above and below $T_{3}=13.96 \mathrm{~K}$. The experimental data (crosses) are in quantitative agreement with the theoretical prediction (solid line) over a broad pressure range except for pressures far away from the sublimation pressure where the theory is not expected to hold. We note that there is no fit parameter involved.

Incomplete wetting occurs for $T<T_{3}$ (Fig. 2a) where the thickness $\ell$ reaches only a value of $18 \AA$ at $P_{0}$. By contrast, for $T>T_{3}$ (Fig. 2b), the film thickness rises steeply as the saturated vapor pressure $P_{0}$ is approached. As can be seen from the theoretical results, this is attributed to the liquid part of the film. In the measurements we cannot distinguish between the solid and the liquid parts of the adsorbed film since the SP technique is sensitive only to total coverage. Furthermore, the measured thickness actually does not strictly diverge at $P_{0}$. Because of experimental limitations we always stay slightly below the chemical potential at liquid-gas coexistence.

At solid-liquid coexistence above $T_{3}$, the total film has a very large thickness indicative of complete wetting. Along the solid-gas coexistence line below $T_{3}$, on the other hand, the film thickness stays finite, its temperature dependence is shown in Fig. 3. Well below $T_{3}$, most of the film is solid with a thickness of only $10 \AA$, corresponding to about 3 monolayers. The increase in thickness upon approaching $T_{3}$ from below, as is obvious in Fig. 3, is not due to growth of the thickness of the solid layer, but of the liquid above it. This is a general feature of triple-point wetting and has been verified experimentally for the system $\mathrm{Ar}$ on $\mathrm{MgO}$ by Rieutord et al. [19]. There is good agreement between

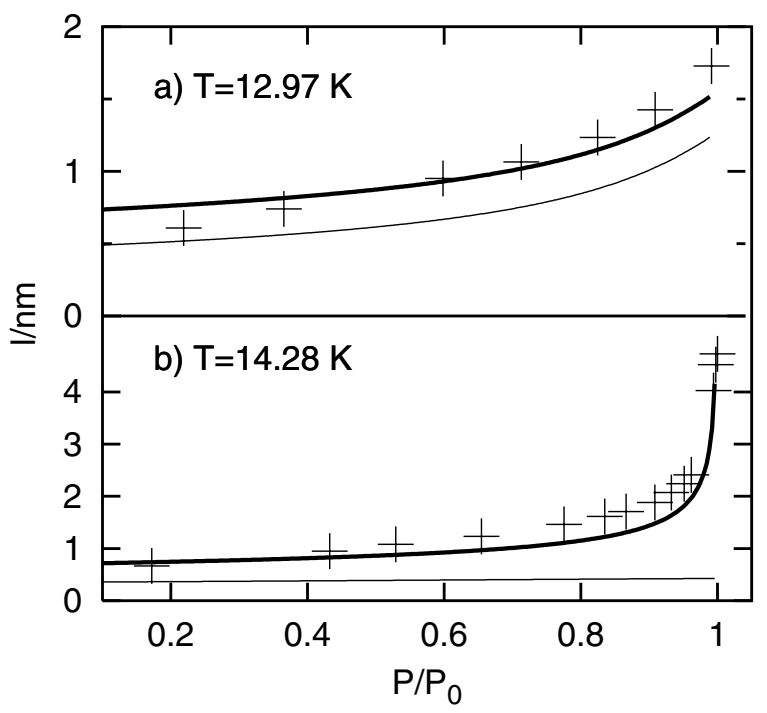

FIG. 2. Thickness $\ell$ of adsorbed hydrogen films on Au substrates as a function of the reduced pressure $P / P_{0}$ for two temperatures (a) $T=12.97 \mathrm{~K}$ (below $T_{3}$ ) and (b) $T=14.28 \mathrm{~K}$ (above $T_{3}$ ). The crosses denote experimental data. The thick lines show theoretical results for the total thickness, and the crystalline part of the adsorbed layer is given by the thin lines. theory and experiment except for temperatures of around $T / T_{3} \approx 0.93$ where a significant part of the adsorbed layer is liquid. This discrepancy is due to the uncertainty in the extrapolation of the liquid-gas coexistence below $T_{3}$ which is required in our theoretical analysis.

We now analyze the hydrogen film thickness as a function of the substrate strength $C$ and compare it to the Gittes-Schick (GS) theory. In Fig. 4, the thickness $\ell$ is shown versus the ratio $R$ of adsorbate-substrate and adsorbate-adsorbate interaction for $T / T_{3}=0.6$. The GS theory yields the solid line diverging at $R=1.88$. A considerable film thickness of around $100 \AA$ (essentially complete wetting) is achieved for $\mathrm{H}_{2}$ on gold where $R=4.5$. However, a finite surface roughness $\bar{G}$ reduces the width considerably, as shown for various degrees of roughness by the dotted and dashed lines in Fig. 4. The reduction in $\ell$ is more pronounced the larger the amplitude of the roughness is. In the following we focus on typical values for $R$ ranging between 2 and 8 and a typical experimental roughness of $\bar{G}=10^{-4} / \sigma^{2}$ (dotted line). In this case the reduction of $\ell$ due to surface roughness can be over many orders of magnitude. This demonstrates that surface roughness rather than lateral strain controls incomplete wetting. As a further qualitative difference, the GS theory yields a thickness which increases with decreasing $R$ while a typical surface roughness leads to a slightly decreasing (though nearly constant) thickness.

We have carried out two sets of additional experiments to test this new interpretation of the origin of triple-point wetting: (i) In order to shift the effective substrate strength to smaller values into the range $R<4.5$ we have studied the adsorption of $\mathrm{H}_{2}$ films on gold surfaces preplated with other noble gases like neon or argon. No substantial increase in the equilibrium $\mathrm{H}_{2}$ film thickness was observed, in contrast to the GS curve in Fig. 4, but in line with the theoretical curve for the substrate roughness studies here. (ii) Another experimental test of the interpretation

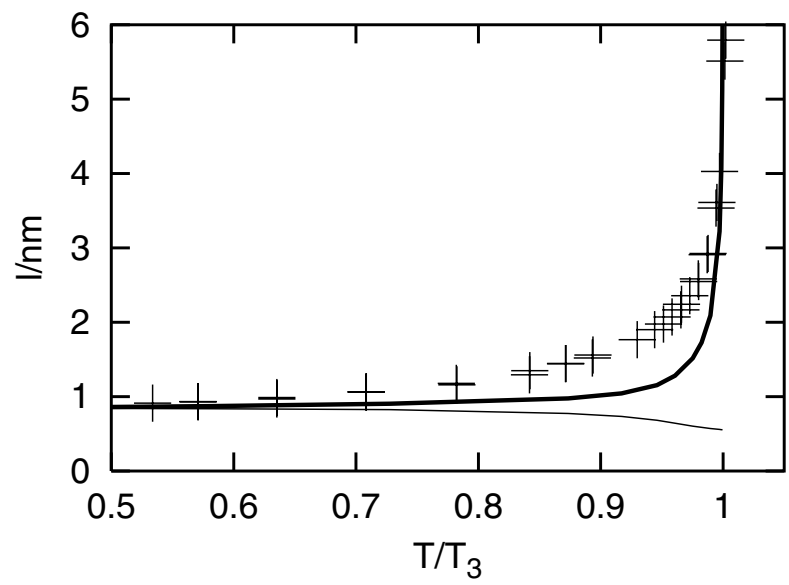

FIG. 3. Thickness $\ell$ of the adsorbed hydrogen film versus temperature along the gas-solid coexistence line. The meaning of the symbols and lines is as described in Fig. 2. 


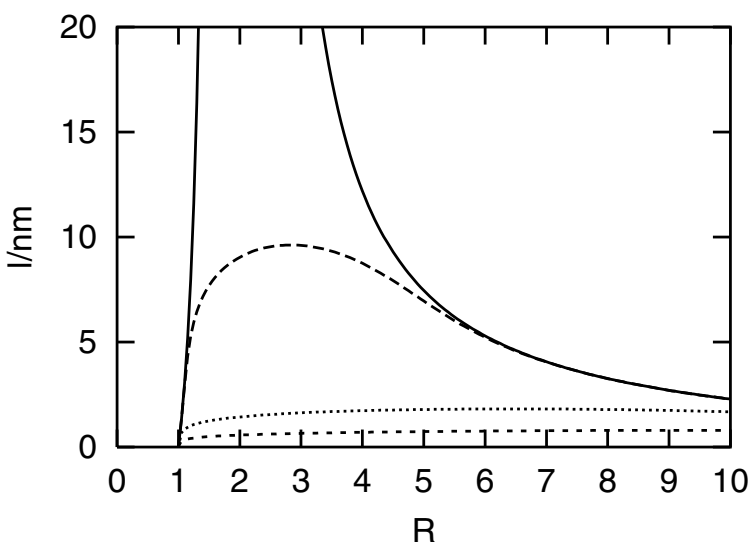

FIG. 4. Thickness $\ell$ of the adsorbed hydrogen layer at solid-gas coexistence for $T / T_{3}=0.6$ as a function of the reduced substrate strength $R$. Solid line: Gittes-Schick theory. The dotted and dashed lines are for different nonvanishing surface roughnesses, $\bar{G}=10^{-2} / \sigma^{2}, \quad \bar{G}=10^{-4} / \sigma^{2}$, and $\bar{G}=10^{-8} / \sigma^{2}$ (bottom to top).

described above is to vary the roughness of the substrate. We have applied a method which allows us to reduce the roughness. It relies on the evaporation of a gold film on a commercial silicon wafer which serves as a quite flat template [20]. The glass prism used as support for the gold film in the SP resonance measurement is glued onto this film, and after removing the silicon wafer the surface of the gold film has essentially the roughness of the wafer. An example of such a profile, determined by AFM, is given in Fig. 1b. The calculation of $\bar{G}$ yields $\bar{G}=2.2 \times 10^{-4} / \sigma^{2}$. Theoretically, this implies an increase of the adsorbed crystalline layer by $15 \%$. In our experiments, we indeed found an increase of $(15 \pm 5) \%$.

In conclusion, we have shown by theory and experiment that incomplete wetting of solid films on substrates is dictated by the surface roughness rather than by the solid strain caused by the substrate attraction. A finite roughness always enforces triple-point wetting. The theory is in good quantitative agreement with our experimental data. As any real substrate is rough, the described scenario plays a decisive role in any wetting situation where solid layers are involved such as for coatings of sculpted substrates or curved nanoparticles [21,22]. Our results imply that extremely smooth substrates are necessary to generate thicknesses of adsorbed van der Waals films larger than $10 \mathrm{~nm}$ which is of direct relevance in such different areas as laser fusion [23], optical spectroscopy [24], surface investigations by slow muons [25], and the determination of the neutrino rest mass [26].
We thank M. Gräfe and C.N. Likos. This work is supported by the Deutsche Forschungsgemeinschaft under Grants No. Le 315/20-1 and No. Lo 418/5-2.

[1] S. Dietrich, in Phase Transitions and Critical Phenomena, edited by C. Domb and J. Lebowitz (Academic Press, London, 1988), Vol. 12, pp. 1-128.

[2] R. Evans, in Liquids at Interfaces, Proceedings of the Les Houches Summer School, Session XLVIII, edited by J. Charvolin, J. F. Joanny, and J. Zinn-Justin (Elsevier, Amsterdam, 1990), pp. 1ff.

[3] H. Gau et al., Science 283, 46 (1999).

[4] K. Kargupta et al., Phys. Rev. Lett. 86, 4536 (2001).

[5] J. Bico et al., Europhys. Lett. 47, 220 (1999).

[6] S. Dietrich and M. Schick, Phys. Rev. B 33, 4952 (1986).

[7] J. L. Seguin et al., Phys. Rev. Lett. 51, 122 (1983); M. Bienfait et al., Phys. Rev. B 29, 983 (1984); J. Krim, J. G. Dash, and J. Suzanne, Phys. Rev. Lett. 52, 640 (1984).

[8] G. Mistura et al., Phys. Rev. Lett. 82, 795 (1999); L. Bruschi and G. Mistura, Phys. Rev. B 61, 4941 (2000); J. Chem. Phys. 114, 1350 (2001).

[9] Y. Qiao and H. K. Christenson, Phys. Rev. Lett. 83, 1371 (1999).

[10] J. Klier et al., Physica (Amsterdam) 284B, 391 (2000).

[11] F. T. Gittes and M. Schick, Phys. Rev. B 30, 209 (1984).

[12] D. B. Pengra et al., Surf. Sci. 245, 125 (1991).

[13] The effects of roughness on the liquid part of the wetting layer is much smaller and can be neglected; see M. Kardar and J. O. Indekeu, Europhys. Lett. 12, 161 (1990); R. R. Netz and D. Andelman, Phys. Rev. E 55, 687 (1997).

[14] These assumptions imply that there are no solid grain boundaries running through the whole crystalline sheet. Local defect formation in the solid near the substrate interface is included in our model as this simply changes $\gamma_{w s}$; see D. A. Huse, Phys. Rev. B 29, 6985 (1984).

[15] L. D. Landau and E. M. Lifshitz, Theory of Elasticity (Pergamon Press, New York, 1986), 3rd ed.

[16] E. Cheng et al., Phys. Rev. B 48, 18214 (1993).

[17] R. Agrawal and D. A. Kofke, Mol. Phys. 85, 43 (1995).

[18] D. J. Quesnel et al., Phys. Rev. B 48, 6795 (1993).

[19] F. Rieutord et al., Europhys. Lett. 37, 565 (1997).

[20] M. Sohaili et al., J. Low Temp. Phys. 122, 249 (2001).

[21] C. Rascón and A. O. Parry, Nature (London) 407, 986 (2000).

[22] M. Heni and H. Löwen, Phys. Rev. Lett. 85, 3668 (2000).

[23] R. S. Craxton et al., Sci. Am. 255, No. 2, 60 (1986).

[24] C. Bressler et al., J. Chem. Phys. 105, 10178 (1996).

[25] E. Morenzoni et al., J. Appl. Phys. 81, 3340 (1997).

[26] See, e.g., L. Fleischmann et al., J. Low Temp. Phys. 119, 615 (2000). 